Sädhanā, Vol. 19, Part 5, October 1994, pp. 765-783. (1) Printed in India.

\title{
Finite element/finite volume approaches with adaptive time stepping strategies for transient thermal problems
}

\author{
RAM V MOHAN and KUMAR K TAMMA* \\ Department of Mechanical Engineering, Institute of Technology, \\ 111 Church Street S.E., University of Minnesota, Minneapolis, \\ MN 55455, USA
}

\begin{abstract}
Transient thermal analysis of engineering materials and structures by space discretization techniques such as the finite element method (FEM) or finite volume method (FVM) lead to a system of parabolic ordinary differential equations in time. These semidiscrete equations are traditionally solved using the generalized trapezoidal family of time integration algorithms which uses a constant single time step. This single time step is normally selected based on the stability and accuracy criteria of the time integration method employed. For long duration transient analysis and/or when severe time step restrictions as in nonlinear problems prohibit the use of taking a larger time step, a single time stepping strategy for the thermal analysis may not be optimal during the entire temporal analysis. As a consequence, an adaptive time stepping strategy which computes the time step based on the local truncation error with a good global error control may be used to obtain optimal time steps for use during the entire analysis. Such an adaptive time stepping approach is described here. Also proposed is an approach for employing combined FEM/FVM mesh partitionings to achieve numerically improved physical representations. Adaptive time stepping is employed thoughout to practical linear/nonlinear transient engineering problems for studying their effectiveness in finite element and finite volume thermal analysis simulations.
\end{abstract}

Keywords. Transient thermal analysis; finite element method; finite volume method; temporal analysis.

\section{Introduction}

The complexity of modern engineering systems places increased demand on thermal analysis of engineering systems with accurate physical interpretations. More and more discrete numerical methods such as the finite difference method (FDM) (Lax \&

\footnotetext{
*For correspondence
} 
Wendroff 1964; Richtmeyer \& Morton 1967), finite element method (FEM) (Zienkiewicz \& Cheung 1965; Bathe 1982; Hughes 1987) etc., are used in conduction heat transfer analysis. The finite element method provides a close approximation of curved boundaries, and a systematic and general way of modelling the boundary conditions. For these reasons, FEM has become a powerful numerical tool in real engineering problems.

The finite difference method is another numerical technique in which the approximate solution is obtained by directly discretizing the governing differential equation. This form of the finite difference method has been historically restricted to rectangular domains and meshes and later been extended to included applicability to general, orthogonal curvilinear coordinate systems. Another approach that has received attention among heat transfer analysts and researchers is the control volume approach (Patankar \& Baliga 1978; Patankar 1980; Baliga \& Patankar 1983). In this approach, an energy balance is applied to discrete control volumes and discretization is used only where surface fluxes require approximation. The property of the control volume approach is that the resulting finite difference equations are conservative, and the discrete equations maintain an accurate accounting of energy flows in the domain. In the application of the finite difference and control volume methods, the coordinate system must be defined over the entire solution domain prior to effecting the discrete method. The finite element method, however, removes the above disadvantage by utilizing a coordinate system which is local to each individual element. Another approach is the use of finite element philosophy (such as isoparametric formulations based on' a finite volume) directly to the conservative energy form of the heat conduction equation, and obtaining the discrete form based on the energy balance. This approach called the finite volume approach, is cited to have the benefits of the finite element method in its applicability to a general curvilinear domain, while preserving the conservation of energy (Schneider 1982; Baliga \& Patankar 1983; Schneider \& Zedan 1983; Banaszek 1984).

The finite element method follows a philosophically different approach than does the finite difference method. In elasticity problems, for e.g., there exists a variational extremum principle such that the minimization of potential energy or the application of the principle of virtual work leads, naturally, to the discrete model. In heat conduction, such a natural formulation with a clear physical interpretation does not exist (Zienkiewicz \& Cheung 1965; Bathe 1982; Owen \& Damjanic 1983; Taylor et al 1983, pp. 405-31; Hughes 1987; Tamma \& Namburu 1989; Namburu \& Tamma 1991), although the existence of variational forms for thermal analysis situations can be proven.

Traditionally, the semi-discrete time dependent equations obtained for the transient analysis are solved with a time stepping scheme using the trapezoidal family of the algorithms (Bathe 1982; Hughes 1987; Tamma \& Namburu 1989; Namburu \& Tamma 1991). Normally a single time step is employed over the entire temporal analysis. This single time step is normally selected based on the stability and accuracy criteria of the time integration scheme used. For a particular trapezoidal family of algorithms, the time step is based on the accuracy desired. Explicit and implicit methods have been used in the past. Whereas explicit methods are easy to code, the severe time step restriction brought by stability considerations have made implicit methods, in particular the trapezoidal Crank-Nicolson method, a logical choice by many analysts. However, although the method is unconditionally stable, the time step is still dictated by accuracy considerations. Nonetheless, the single time step 


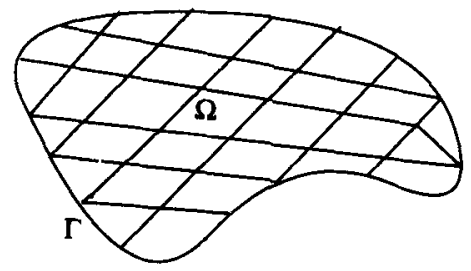

Figure 1. Typical solution domain.

selected initially for desired accuracy levels may not be optimal at all times during the analysis. It is possible that the time step selected may be too large for the accuracy desired in the analysis. It is also possible that the initial time step selected is too small during the analysis and the same desired accuracy could have been obtained using a larger time step during the analysis. An adaptive automated time stepping approach based on local truncation error with good control of global error will yield optimal time steps which changes continually during the analysis. Such an adaptive time stepping strategy based on local truncation error is considered here for the practical applicability to engineering problems in conjunction with finite element and finite volume methods. Conduction/convection/radiation effects are included. A brief overview of finite element/finite volume methods is first described followed by an effective automated time stepping approach adopted in this paper. Combined mesh partitionings involving FEM/FVM meshes based on physical situations to obtain numerically improved physical representations have been considered. Numerical test cases are described and comparative pros and cons are identified for practical situations.

\section{Geometry and element definition}

For purposes of illustration, attention is restricted here to two-dimensional problems. A typical solution domain is shown in figure 1 which is subdivided into linear quadrilateral finite elements. A typical element is shown in figure 2, with the local coordinate system $(\xi, \eta)$. For the individual element, the local node numbers ranging from 1 through 4 are shown and the temperature field $T$ and the global coordinates

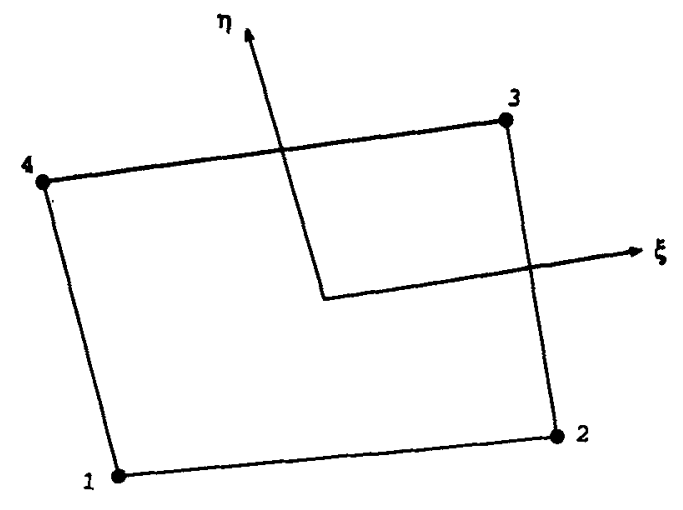

Figure 2. A typical finite volume element. 
$x, y$ are interpolated using bilinear isoparametric formulations of the form

$$
\begin{aligned}
& T=\sum_{i=1}^{4} N_{i} T_{i}, \\
& x=\sum_{i=1}^{4} N_{i} x_{i}, \\
& y=\sum_{i=1}^{4} N_{i} y_{i}
\end{aligned}
$$

where the shape functions $N_{i}$ are defined by

$$
\begin{aligned}
& N_{1}=\frac{1}{4}(1-\xi)(1-\eta), \\
& N_{2}=\frac{1}{4}(1-\eta)(1+\xi), \\
& N_{3}=\frac{1}{4}(1+\xi)(1+\eta), \\
& N_{4}=\frac{1}{4}(1+\eta)(1-\xi) .
\end{aligned}
$$

The $x$ and $y$ derivatives of the temperature can be determined as

$$
B=\left\{\begin{array}{l}
\frac{\partial T}{\partial x}=\sum_{i=1}^{4} \frac{\partial N_{i}}{\partial x} T_{i} \\
\frac{\partial T}{\partial y}=\sum_{i=1}^{4} \frac{\partial N_{i}}{\partial y} T_{i}
\end{array}\right\}
$$

where the $x$ and $y$ derivatives of the shape functions appearing in (5) are determined by

$$
\left\{\begin{array}{l}
\frac{\partial N_{i}}{\partial x} \\
\frac{\partial N_{i}}{\partial y}
\end{array}\right\}=\frac{1}{\operatorname{Det}[J]}\left[\begin{array}{rrr}
\frac{\partial y}{\partial \eta} & -\frac{\partial y}{\partial \xi} & \frac{\partial N_{i}}{\partial \xi} \\
-\frac{\partial x}{\partial \eta} & \frac{\partial x}{\partial \xi} & \frac{\partial N_{i}}{\partial \eta}
\end{array}\right]
$$

Considering a general line segment shown in figure 3 , the normal vector while traversing along from point 1 to point 2 is defined by

$$
\mathrm{d} \mathbf{S}=\mathrm{d} y \hat{i}-\mathrm{d} x \hat{j}
$$

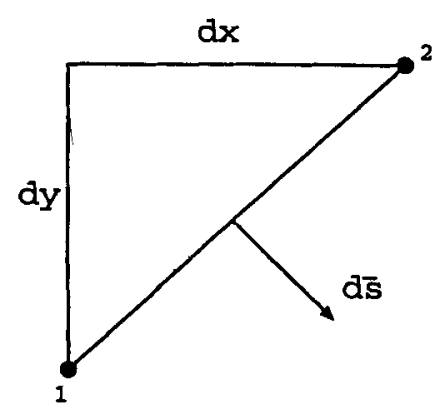

Figure 3. Typical surface line segment. 
The above definitions set the basis in the development of the finite element/finite volume formulations as described next.

Finite element equations and element matrices

Of interest here are general nonlinear transient thermal problems governed by

$$
\rho_{c}(\partial T / \partial t)-\nabla \cdot(K \nabla T)=Q
$$

with the appropriate boundary and initial conditions. These are defined as

where

$$
\begin{array}{ll}
T=T_{p}, & \text { on } \Gamma_{1}, \\
q_{i} n_{i}-q_{s}+q_{h}+q_{r}=0, & \text { on } \Gamma_{2},
\end{array}
$$

$$
\begin{aligned}
& q_{h}=h\left(T-T_{h}\right), \\
& q_{r}=\sigma \varepsilon\left(T^{4}-T_{r}^{4}\right) .
\end{aligned}
$$

Applying the traditional Galerkin finite element method to (8-12), using the shape functions defined in the earlier section, leads to a semi-discrete equation of the form

$$
[C]\{\dot{T}\}+\left[\left[K_{c}\right]+\left[K_{h}\right]+\left[K_{r}\right]\right]\{T\}=\left\{R_{c}\right\}+\left\{R_{Q}\right\}+\left\{R_{q}\right\}+\left\{R_{h}\right\}+\left\{R_{r}\right\}
$$

where $[C]$ is the capacitance matrix, $\left[\dot{K}_{c}\right],\left[K_{h}\right]$ and $\left[K_{r}\right]$ are element conduction matrices corresponding to conduction, convection and radiation respectively; $\left\{R_{c}\right\}$, $\left\{R_{Q}\right\},\left\{R_{q}\right\},\left\{R_{h}\right\},\left\{R_{r}\right\}$ are the load vectors from specified nodal temperatures, internal heat generation, edge heating, radiation and convection. These are defined as

$$
\begin{aligned}
{[C] } & =\int_{\Omega_{e}} \rho c N^{T} N \mathrm{~d} \Omega, \\
{\left[K_{c}\right] } & =\int_{\Omega_{e}} B^{T} K B \Omega, \\
{\left[K_{h}\right] } & =\int_{\Gamma_{2}} h N^{T} N \mathrm{~d} \Omega, \\
{\left[K_{r}\right]\{T\} } & =\int_{\Gamma_{2}} \sigma \varepsilon T^{4} N^{T} \mathrm{~d} \Omega, \\
\left\{R_{c}\right\} & =-\int_{\Gamma_{1}}(\mathbf{q} \cdot \hat{n}) N^{T} \mathrm{~d} \Gamma, \\
\left\{R_{Q}\right\} & =\int_{\Omega_{e}} Q N^{T} \mathrm{~d} \Omega, \\
\left\{R_{q}\right\} & =\int_{\Gamma_{2}} q_{s} N^{T} \mathrm{~d} \Gamma, \\
\left\{R_{h}\right\} & =\int_{\Gamma_{2}} h T_{h} N^{T} \mathrm{~d} \Gamma,
\end{aligned}
$$




$$
\left\{R_{r}\right\}=\int_{\Gamma_{2}} \sigma \varepsilon T_{r}^{4} N^{T} \mathrm{~d} \Gamma,
$$

with initial conditions $\mathbf{T}(0)=\mathbf{T}_{0}$.

\section{Finite volume equations and element matrices}

Here a procedure analogous to that followed by the conventional finite element formulation is considered. In this a single, isolated finite volume is first considered. The application of an energy balance to this element gives rise to element level matrices, which are related to the nodal temperature values of the element. Once the appropriate capacitance and conduction and load formulations are obtained, assembly rules in a similar sense of the finite element formulations can be employed to construct the global equation system from the element level equations.

The element matrices are constructed using a single element as shown in figure 4. The single finite volume element is subdivided into four internal finite volumes, each of which is associated with the corresponding nearest neighboring node of the element. In the linear quadrilateral element shown in figure 4, the control volume boundaries are chosen to be coincident with the element exterior boundaries and with the local coordinate surfaces defined by $\xi=0$ and $\eta=0$. This choice is consistent from element to element in the entire formulation, and this boundary selection makes the evaluation of the integrals defined in the formulation easier.

The energy balance is now applied to one such control volume and is expressed as: net rate of conduction into control volume $=$ rate of generation within control volume + rate of change of energy within the control volume.

For the control volume associated with node 3 (sub-control volume 3 ) in figure 4, this energy balance can be mathematically expressed as

$$
Q_{2,3}+Q_{4,3}+Q_{\mathrm{e} 1,3}+Q_{\mathrm{e} 2,3}+\iint_{c V} Q \mathrm{~d} V=\frac{\partial}{\partial t} \iint_{C V} \rho c T \mathrm{~d} V
$$

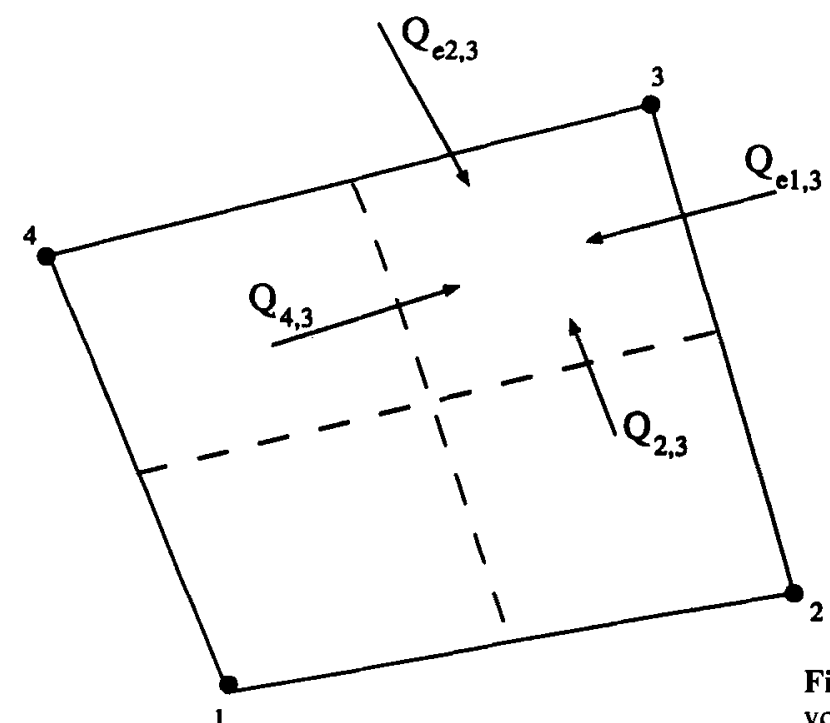

Figure 4. Single element with finite volume subdivision and heat flows. 
where the limits of the integral correspond to the control volume associated with the node 3 . The subscripts $e 1$ and $e 2$ in (23) refer to the energy flows into control volume 3 through surfaces which are on the exterior of the element and arise either from the physical domain boundary or from adjacent elements. In the case of adjacent elements, the heat fluxes cancel with each other, while in the case of the boundary, the boundary conditions determine the contribution of these terms. The interior terms which give rise to the conduction matrix coefficients are now considered.

In general, the heat flow through a surface can be expressed as

$$
Q=\int_{S} \mathbf{q} \cdot \mathrm{d} \mathbf{S}
$$

where the heat flux vector $\mathbf{q}$ is given as

$$
\mathbf{q}=-k_{x} \frac{\partial T}{\partial x} \hat{i}-k_{y} \frac{\partial T}{\partial y} \hat{j} .
$$

In this case

$$
\mathrm{d} \mathbf{S}=\mathrm{d} y \hat{i}-\mathrm{d} x \hat{j} .
$$

Based on this the various matrices for the finite volume element can be obtained. The conduction contributions are

$$
\begin{aligned}
& K_{1, i}=\left.\int_{\xi=-1}^{\xi=0}\left(k_{x} \frac{\partial N_{i}}{\partial x} \frac{\partial y}{\partial \xi}-k_{y} \frac{\partial N_{i}}{\partial y} \frac{\partial x}{\partial \xi}\right)\right|_{\eta=0} \mathrm{~d} \xi+ \\
& K_{2, i}=\left.\int_{\xi=1}^{\eta=0}\left(-k_{x} \frac{\partial N_{i}}{\partial x} \frac{\partial y}{\partial \eta}+k_{y} \frac{\partial N_{i}}{\partial x} \frac{\partial x}{\partial \eta}\right)\right|_{\xi=0} \mathrm{~d} \eta \\
& K_{3, i}=\left.\int_{\eta=0}^{\eta=1}\left(k_{x} \frac{\partial N_{i}}{\partial x} \frac{\partial y}{\partial \xi}-k_{y} \frac{\partial N_{i}}{\partial y} \frac{\partial x}{\partial \xi}\right)\right|_{\eta=0} \mathrm{~d} \xi+ \\
& \left.k_{\eta=-1}^{\eta} \frac{\partial N_{i}}{\partial x} \frac{\partial y}{\partial \eta}-k_{y} \frac{\partial N_{i}}{\partial y} \frac{\partial x}{\partial \eta}\right)\left.\right|_{\xi=0} \mathrm{~d} \eta+ \\
& \left.\int_{\xi=0}^{\xi=1}\left(-k_{x} \frac{\partial N_{i}}{\partial x} \frac{\partial y}{\partial \xi}+k_{y} \frac{\partial N_{i}}{\partial x} \frac{\partial x}{\partial \xi}\right)\right|_{\eta=0} \mathrm{~d} \xi \\
& K_{4, i}=\left.\int_{\eta=0}^{\eta=1}\left(-k_{x} \frac{\partial N_{i}}{\partial x} \frac{\partial y}{\partial \eta}+k_{y} \frac{\partial N_{i}}{\partial y} \frac{\partial x}{\partial \eta}\right)\right|_{\xi=0} \mathrm{~d} \eta+ \\
& \left.\int_{\xi=0}^{\xi=0}\left(-k_{x} \frac{\partial N_{i}}{\partial x} \frac{\partial y}{\partial \xi}+k_{y} \frac{\partial N_{i}}{\partial x} \frac{\partial x}{\partial \xi}\right)\right|_{\eta=0} \mathrm{~d} \xi
\end{aligned}
$$

where $i=1,4$ in the above expressions are for a bilinear element.

Based on the energy balances of each of the control volumes, the capacitance matrix entries are given by the following expressions

$$
C_{1, i}=\int_{\xi=-1}^{\xi=0} \int_{\eta=-1}^{\eta=0} \rho c N_{i} \operatorname{Det}[J] \mathrm{d} \xi \mathrm{d} \eta,
$$




$$
\begin{aligned}
& C_{2, i}=\int_{\xi=0}^{\xi=1} \int_{\eta=-1}^{\eta=0} \rho c N_{i} \operatorname{Det}[J] \mathrm{d} \xi \mathrm{d} \eta, \\
& C_{3, i}=\int_{\xi=0}^{\xi=1} \int_{\eta=0}^{\eta=1} \rho c N_{i} \operatorname{Det}[J] \mathrm{d} \xi \mathrm{d} \eta, \\
& C_{4, i}=\int_{\xi=-1}^{\xi=0} \int_{\eta=0}^{\eta=1} \rho c N_{i} \operatorname{Det}[J] \mathrm{d} \xi \mathrm{d} \eta .
\end{aligned}
$$

The stiffness matrix corresponding to surface convection is given by

$$
\begin{aligned}
& \left(K_{h}\right)_{1, i}=\int_{\xi=-1}^{\xi=0} \int_{\eta=-1}^{\eta=0} h N_{i} \operatorname{Det}[J] \mathrm{d} \xi \mathrm{d} \eta, \\
& \left(K_{h}\right)_{2, i}=\int_{\xi=0}^{\xi=1} \int_{\eta=-1}^{\eta=0} h N_{i} \operatorname{Det}[J] \mathrm{d} \xi \mathrm{d} \eta, \\
& \left(K_{h}\right)_{3, i}=\int_{\xi=0}^{\xi=1} \int_{\eta=0}^{\eta=1} h N_{i} \operatorname{Det}[J] \mathrm{d} \xi \mathrm{d} \eta, \\
& \left(K_{h}\right)_{4, i}=\int_{\xi=-1}^{\xi=0} \int_{\eta=0}^{\eta=1} h N_{i} \operatorname{Det}[J] \mathrm{d} \xi \mathrm{d} \eta .
\end{aligned}
$$

The stiffness matrices corresponding to the surface radiation involved in the Jacobian for the nonlinear formulations are given by

$$
\begin{aligned}
& \left(K_{R}\right)_{1, i}=\int_{\xi=-1}^{\xi=0} \int_{\eta=-1}^{\eta=0} 4 \sigma \varepsilon T_{\mathrm{ave}}^{3} N_{i} \operatorname{Det}[J] \mathrm{d} \xi \mathrm{d} \eta, \\
& \left(K_{R}\right)_{2, i}=\int_{\xi=0}^{\xi=1} \int_{\eta=-1}^{\eta=0} 4 \sigma \varepsilon T_{\mathrm{ave}}^{3} N_{i} \operatorname{Det}[J] \mathrm{d} \xi \mathrm{d} \eta, \\
& \left(K_{R}\right)_{3, i}=\int_{\xi=0}^{\xi=1} \int_{\eta=0}^{\eta=1} 4 \sigma \varepsilon T_{\mathrm{ave}}^{3} N_{i} \operatorname{Det}[J] \mathrm{d} \xi \mathrm{d} \eta, \\
& \left(K_{R}\right)_{4, i}=\int_{\xi=-1}^{\xi=0} \int_{\eta=0}^{\eta=1} 4 \sigma \varepsilon T_{\mathrm{ave}}^{3} N_{i} \operatorname{Det}[J] \mathrm{d} \xi \mathrm{d} \eta .
\end{aligned}
$$

The various volume load vectors for volume heating, convection and radiation are given by the following integral expressions.

For the volumetric heat source $Q$, the corresponding load vectors are given by

$$
\begin{aligned}
& \left(R_{Q}\right)_{1}=\int_{\xi=-1}^{\xi=0} \int_{\eta=-1}^{\eta=0} Q \operatorname{Det}[J] \mathrm{d} \xi \mathrm{d} \eta, \\
& \left(R_{Q}\right)_{2}=\int_{\xi=0}^{\xi=1} \int_{\eta=-1}^{\eta=0} Q \operatorname{Det}[J] \mathrm{d} \xi \mathrm{d} \eta, \\
& \left(R_{Q}\right)_{3}=\int_{\xi=0}^{\xi=1} \int_{\eta=0}^{\eta=1} Q \operatorname{Det}[J] \mathrm{d} \xi \mathrm{d} \eta,
\end{aligned}
$$




$$
\left(R_{Q}\right)_{4}=\int_{\xi=-1}^{\xi=0} \int_{\eta=0}^{\eta=1} Q \operatorname{Det}[J] \mathrm{d} \xi \mathrm{d} \eta .
$$

The load vectors due to surface convection are given by

$$
\begin{aligned}
& \left(R_{h}\right)_{1}=\int_{\xi=-1}^{\xi=0} \int_{\eta=-1}^{\eta=0} h T_{h} \operatorname{Det}[J] \mathrm{d} \xi \mathrm{d} \eta \\
& \left(R_{h}\right)_{2}=\int_{\xi=0}^{\xi=1} \int_{\eta=-1}^{\eta=0} h T_{h} \operatorname{Det}[J] \mathrm{d} \xi \mathrm{d} \eta \\
& \left(R_{h}\right)_{3}=\int_{\xi=0}^{\xi=1} \int_{\eta=0}^{\eta=1} h T_{h} \operatorname{Det}[J] \mathrm{d} \xi \mathrm{d} \eta, \\
& \left(R_{h}\right)_{4}=\int_{\xi=-1}^{\xi=0} \int_{\eta=0}^{\eta=1} h T_{h} \operatorname{Det}[J] \mathrm{d} \xi \mathrm{d} \eta,
\end{aligned}
$$

where $T_{h}$ is the ambient temperature. The load vectors due to surface radiation are given by

$$
\begin{aligned}
& \left(R_{r}\right)_{1}=\int_{\xi=-1}^{\xi=0} \int_{\eta=-1}^{\eta=0} \sigma \varepsilon T_{h}^{4} \operatorname{Det}[J] \mathrm{d} \xi \mathrm{d} \eta \\
& \left(R_{r}\right)_{2}=\int_{\xi=0}^{\xi=1} \int_{\eta=-1}^{\eta=0} \sigma \varepsilon T_{h}^{4} \operatorname{Det}[J] \mathrm{d} \xi \mathrm{d} \eta \\
& \left(R_{r}\right)_{3}=\int_{\xi=0}^{\xi=1} \int_{\eta=0}^{\eta=1} \sigma \varepsilon T_{h}^{4} \operatorname{Det}[J] \mathrm{d} \xi \mathrm{d} \eta \\
& \left(R_{r}\right)_{4}=\int_{\xi=-1}^{\xi=0} \int_{\eta=0}^{\eta=1} \sigma \varepsilon T_{h}^{4} \operatorname{Det}[J] \mathrm{d} \xi \mathrm{d} \eta
\end{aligned}
$$

The element matrices due to edge heating, edge convection and edge radiation are given by the line integrals. For example for the control volume 3 it is given by an integral of the form

$$
\left(K_{\text {edge }}\right)_{3, i}=\int_{\eta=0}^{\eta=1} N_{i}(1, \eta) \operatorname{Det}[S] \mathrm{d} \eta,
$$

where $\operatorname{Det}[S]$ is the determinant associated with the edge integral. For a typical edge of length $l$ the associated stiffness matrix form is given by

$$
K_{\text {edge }}=\frac{l}{2}\left[\begin{array}{cc}
\frac{1}{4} & \frac{3}{4} \\
\frac{3}{4} & \frac{1}{4}
\end{array}\right]
$$

The load vector contribution due to the heating, radiation and convection effects on an edge is given by a vector of the form

$$
R_{\text {edge }}=\frac{l}{2}\left[\begin{array}{l}
1 \\
1
\end{array}\right]
$$




\section{Adaptive time integration methods}

An automated adaptive time stepping approach is employed here for both the finite element method (FEM) and the finite volume method. This adaptive time stepping approach based on the localized error control which also gives a good global error control has been used in conjunction with the generalized trapezoidal $\alpha$-family of time integration methods (Bathe 1982; Hughes 1987) used for the semi-discrete heat equation. For completeness, the generalized trapezoidal family of algorithms is briefly described followed by the adaptive time stepping strategy.

\section{Generalized trapezoidal family method}

The semidiscrete heat equation can be written as

$$
\mathbf{C} \dot{\mathbf{T}}+\mathbf{K T}=\mathbf{F},
$$

where $\mathbf{C}$ is the capacitance matrix, $\mathbf{K}$ is the conduction matrix, $\mathbf{F}$ is the heat supply vector, $\mathbf{T}$ is the temperature vector, and $\mathbf{T}$ is the time derivative of $\mathbf{T}$. The matrices $\mathbf{C}$ and $\mathbf{K}$ are assumed to be symmetric. The heat supply is a prescribed function of $t$ and $T$ comes from the various load vector terms considered earlier.

The initial-value problem consists of finding a function $\mathbf{T}$ satisfying (58) and

$$
\mathbf{T}(0)=\mathbf{T}_{0},
$$

where $\mathbf{T}_{0}$ is initially given.

The most well known and commonly used algorithms for solving (58) are members of the generalized trapezoidal family of methods, which consists of the following equations

$$
\begin{aligned}
\mathbf{C} \dot{\mathbf{T}}_{n+1}+\mathbf{K} \mathbf{T}_{n+1} & =\mathbf{F}_{n+1}, \\
\mathbf{T}_{n+1} & =\mathbf{T}_{n}+\Delta t \dot{\mathbf{T}}_{n+\alpha}, \\
\dot{\mathbf{T}}_{n+\alpha} & =(1-\alpha) \dot{\mathbf{T}}_{n}+\alpha \dot{\mathbf{T}}_{n+1},
\end{aligned}
$$

where $\mathbf{T}_{n}$ and $\dot{\mathbf{T}}_{n}$ are the approximations to $\mathbf{T}\left(t_{n}\right)$ and $\dot{\mathbf{T}}\left(t_{n}\right)$ respectively; $\mathbf{F}_{n+1}=\mathbf{F}\left(t_{n+1}\right) ; \Delta t$ is the time step, assumed constant for the time being; and $\alpha$ is the parameter in the interval $[0,1]$ where $\alpha=0$ corresponds to the forward Euler scheme; $\alpha=1 / 2$ is the Crank-Nicholson scheme; $\alpha=2 / 3$ is the Galerkin scheme and $\alpha=1$ is the backward Euler scheme. Of these schemes, only the $\alpha=1 / 2$ is second order accurate in time.

Customarily most of the approaches adopted for the time integration have been based on a single time step being used in the entire analysis. This time step is normally selected based on the stability and accuracy criteria which depends on the value of $\alpha$. In particular, in the finite volume method the value of $\alpha=1$ has been extensively used which corresponds to the backward Euler method which is unconditionally stable, but only first order accurate. In the finite element method, however, $\alpha=1 / 2$ and $\alpha=0$ have been the more prominent approaches for transient thermal analysis simulations. 


\section{Adaptive time stepping strategy}

The finite element/finite volume methods traditionally employ a single time stepping strategy in conjunction with the selected generalized trapezoidal family of schemes described earlier. In this case the time step $\Delta t$ selected for the time stepping in based on the accuracy desired. Instead of a fixed time step it is desirable to have an adaptive automated time step which varies continually during the analysis minimizing the local truncation error and with a good global error control. Such an approach will give optimal time steps with a good error control. Eriksson and Johnson (Eriksson \& Johnson 1987; Johnson 1988; Thomea et al 1990) developed error bounds for a class of problems defined by the first-order parabolic differential equations to which the semi-discrete form of the heat equation belongs. The error bound can be written (with particular reference to the backward Euler) as

$$
\max _{t \leqslant t_{N}}\left\|\mathbf{T}\left(t_{n}\right)-\mathbf{T}_{n}\right\| \leqslant C\left[\log \left(t_{N} / \Delta t_{n}\right)+1\right]^{1 / 2} \max _{n \leqslant N}\left(\Delta t_{n} \max _{t \in I_{n}}\|\mathbf{T}(t)\|\right) .
$$

The adaptive time stepping approach is based on the following strategy. Suppose, $\delta$ is a given tolerance and the discrete equation for $\mathbf{T}$ satisfies

$$
\max _{t \leqslant t_{N}}\left\|\mathbf{T}(t)-\mathbf{T}_{n}\right\| \leqslant \delta
$$

Using (63) and neglecting the logarithmic error, the time step $\Delta t_{n}$ can be chosen such that

$$
C \Delta t_{n} \max _{t \leqslant t_{N}}\|\dot{\mathbf{T}}(t)\| \leqslant \delta
$$

where the constant $C$ is known approximately. Since $\mathbf{T}(t)$ is not known, the condition given by (65) is replaced by

$$
\left\|\mathbf{T}_{n}-\mathbf{T}_{n-1}\right\| \leqslant \delta / C=\Delta T_{t o l} .
$$

This leads to an algorithm for $\Delta t_{n}$, assuming $\mathbf{T}_{n-1}$ has already been computed. This permits an adaptive time stepping strategy as follows:

(1) choose the initial step size $\Delta t_{0}\left(\Delta t_{n-1}\right)$;

(2) increment in time with $\Delta t_{n}=\Delta t_{n-1}$ to obtain the solution $\mathbf{T}_{n}$;

(3) if $\delta / \gamma C \leqslant\left\|\mathbf{T}_{n}-\mathbf{T}_{n-1}\right\| \leqslant \delta / C$;

(4) then stop and accept the time step $\Delta t_{n}$. Otherwise increase or decrease $\Delta t_{n}$ by a factor, say, 2 (for example).

Here $\delta$ is the required selected tolerance, $C$ is an assumed known constant, $\gamma$ is a suitable constant ( 2 or 3 ). $\|\cdots\|$ refers to the $L_{\infty}$ norm. This gives a simple error estimator with a good control of global error.

The estimated error employing the above is thus

$$
\max _{t \leqslant t_{N}}\left\|\mathbf{T}_{\text {exact }}(t)-\mathbf{T}(t)\right\| \leqslant \delta / C=\Delta T_{t o l}
$$


That is,

$$
\left\|T_{\text {exact }}(t)-\mathbf{T}(t)\right\| \leqslant \delta
$$

The above adaptive time stepping strategy is theoretically justified for nonlinear parabolic problems (Eriksson \& Johnson 1987; Thomea et al 1990) and can be used in heat transfer situations and solidification problems (Ouyang \& Tamma 1992). The additional computations involved in this time step control are considerably small. Limitations on permissible time steps are based on stability for the explicit form of the time integration and based on accuracy for the implicit form of the time integration.

For the adaptive time stepping strategy, $\Delta T_{\text {tol }}$ can also be automatically selected to control the error employing the following proposed procedure. At step $n$.

$$
\text { - Set } \Delta T_{t o l}=x \% \text { of } T_{r} \text { where } T_{r}=\left|\mathbf{T}_{\max }-\mathbf{T}_{\min }\right| \text {. }
$$

This adaptive time stepping strategy based on a posteriori error estimates, is reliable and for $\left\|\mathbf{T}_{\text {exact }}-\mathbf{T}\right\|<\delta$ the error is bounded. The above-mentioned approach enables control of error and permits an effective and optimal strategy for the numerical solution of both linear and nonlinear heat transfer problems, employing finite element and finite volume approaches.

The time step selected during the time advancement based on the above scheme has been used in test problems involving only finite element/finite volume element formulations for general heat transfer problems. Comparisons are drawn for the pros/cons over single time stepping strategies.

For the adaptive time stepping strategy, additional computations at intermediate levels are necessary whenever there is a change in the time step. Caution should be exercised to adjust the time steps when they become too small or too large, and when there are rapid transient fluctuations.

\section{Test problems}

To validate the present developments the following test problems are considered. These test problems with various material nonlinearities and radiation effects cover a wide range of potential problems encountered in engineering practice. For the transient thermal analysis the time integration has been performed using the generalized trapezoidal $\alpha$ family of algorithms, in conjunction with both single and adaptive time stepping strategies. Finite element and finite volume based elements are used as appropriate and an illustrative mesh partitioning example is also described. All computations have been performed on Cray XMP. The test examples are described next.

\section{Transient nonlinear analysis of the space shuttle thermal protection system}

The problem involves one-dimensional representations of the thermal protection system (TPS) of the space shuttle which is composed of different materials (figure 5). The thermophysical properties of various materials are given in figure 6 . The shuttle TPS, which is initially at $T=322 \mathrm{~K}$ is subjected to a sudden step heat flow input at the exposed surface as shown in Figure 5. The exposed surface of the TPS is assumed 


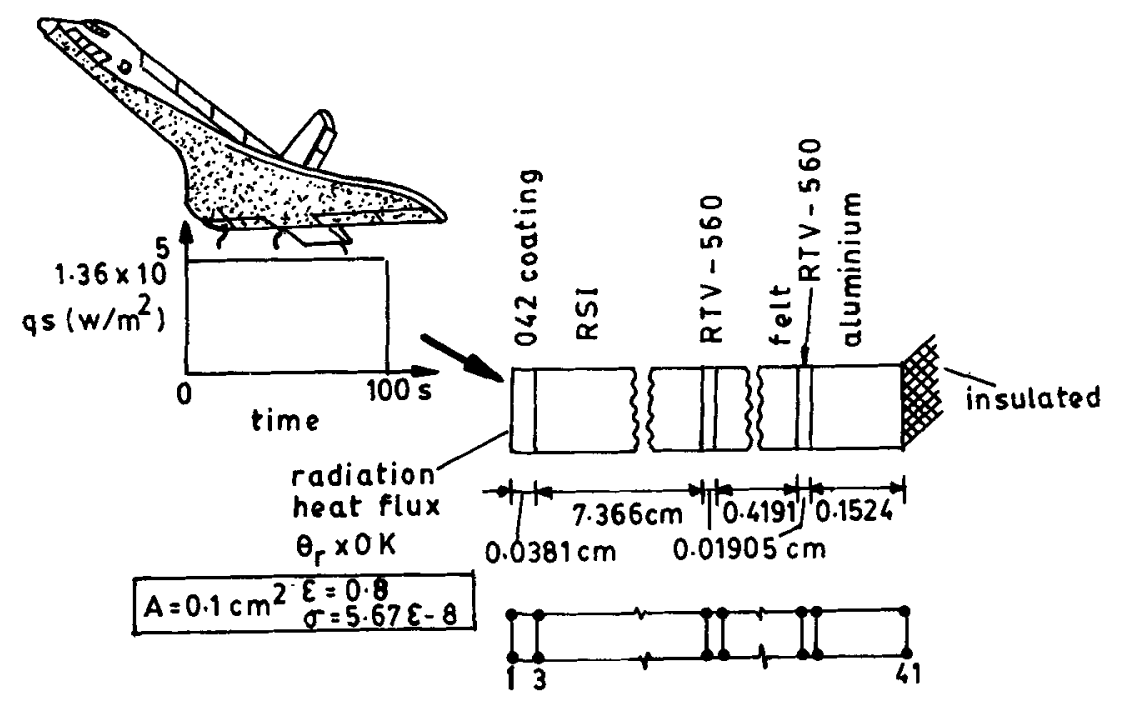

Figure 5. Shuttle TPS and description of the problem.
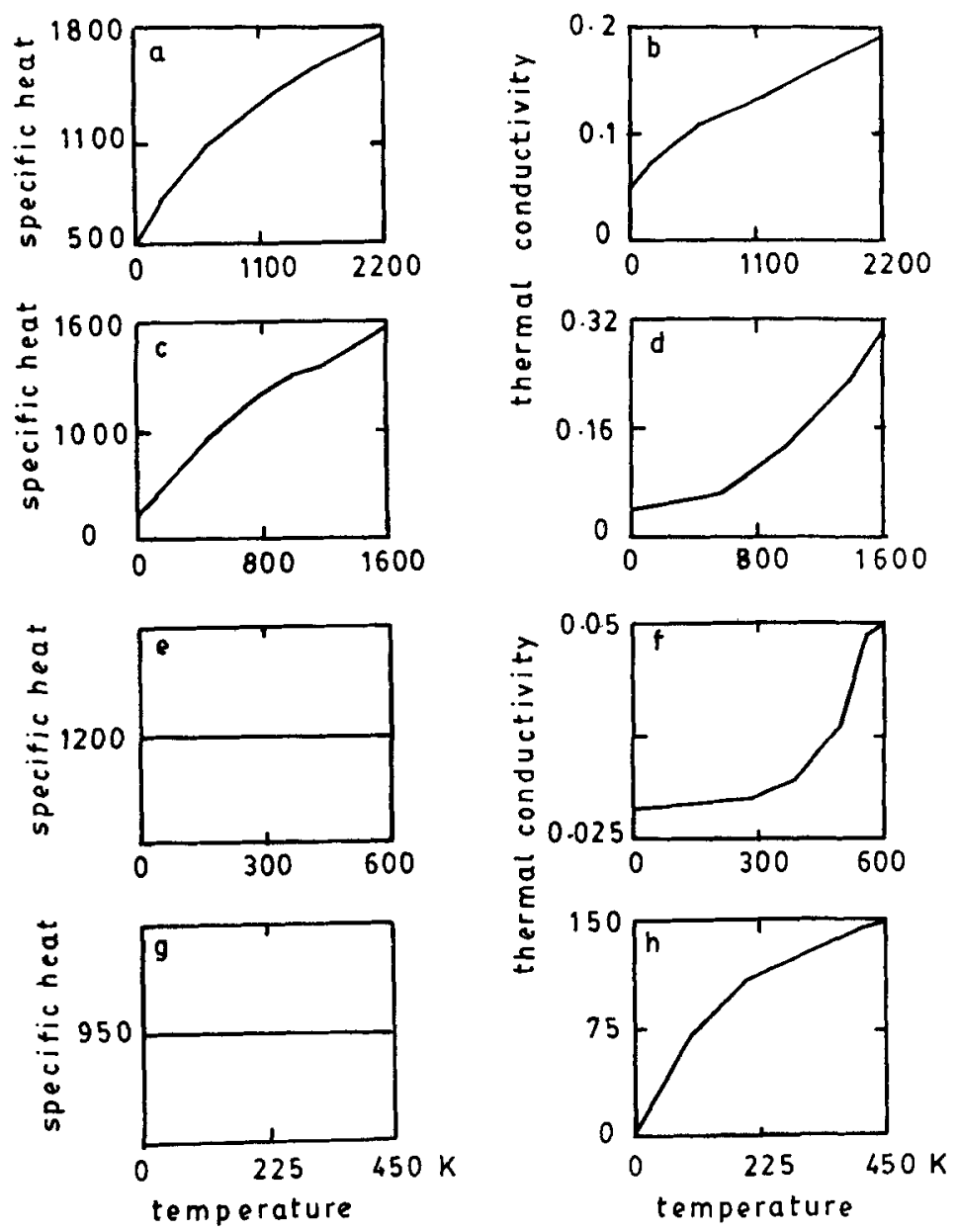

Figure 6. Thermal properties of various materials. $C_{p}$ and $K$ respectively for 042 (a \& b), RSI (c \& d), felt (e \& f), and aluminum ( $\&$ h). 


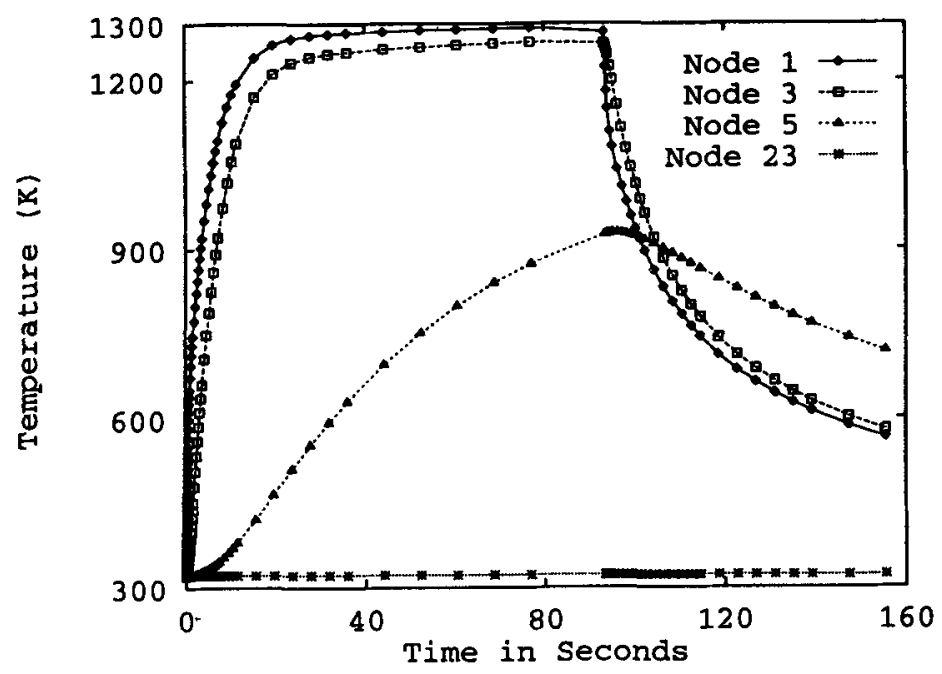

Figure 7. Temperature histories of various locations of TPS.

to radiate to an ambient absolute zero temperature continuously. The same problem was also analysed by Williams \& Curry (1977), and Namburu \& Tamma (1991). For evaluating the effectiveness of the finite volume formulations, a total of 19 finite volume elements with a total of 40 d.o.f. has been employed. A typical transient thermal response is shown in figure 7 . The results obtained employing adaptive time stepping and without adaptive time stepping and using the Galerkin finite elements and finite volume elements show good comparison. The adaptive time stepping variations during the analysis for both the Galerkin finite element method and finite volume method are shown in figure 8 . It is clear from the figure that the time step

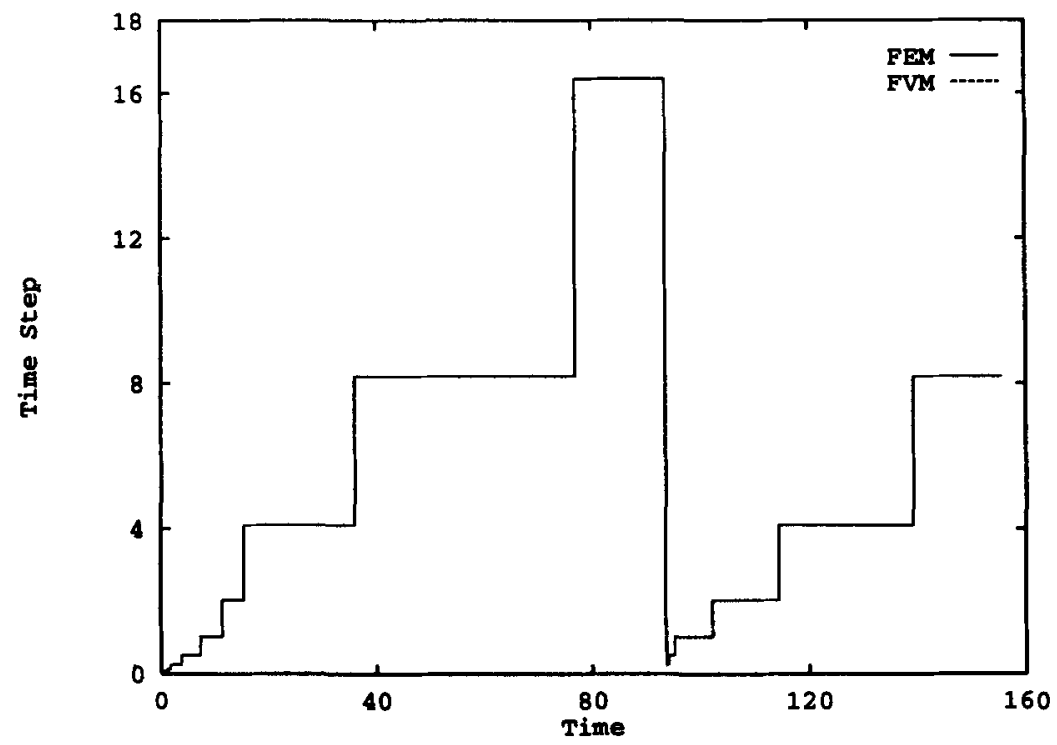

Figure 8. Adaptive time step variations. 
has adapted based on a posteriori error estimator and the adaptivity is clearly seen when there are sudden changes in the external heat fluxes etc., as seen when the global time is 100 seconds. A time step of 0.01 seconds ( $\alpha=0.5$, extremely conservative) was employed to serve as a benchmark result without adaptive time stepping. With the adaptive time stepping procedure employed, the analysis was completed in approximately 200 steps for the same time period and the results are in excellent agreement.

\section{Two-dimensional rectangular plate with convection and radiation along two edges}

In this example a rectangular plate with nonlinear boundary conditions involving radiation and nonlinear material properties is considered. One quarter of the plate is modelled due to symmetry. The physical plate and the finite element model are shown in figure 9. The nonlinear material properties are as shown in figure 10. Comparison of results with traditional finite element formulation shows the effectiveness of the finite volume method as shown in figure 11. A time step of 0.05 seconds $(\alpha=0.5)$ is employed when the analysis is done without resorting to adaptive time stepping which requires 200 steps, and with adaptive time stepping the analysis is completed in about 120 steps. The adaptive time step variations are shown in figure 12.

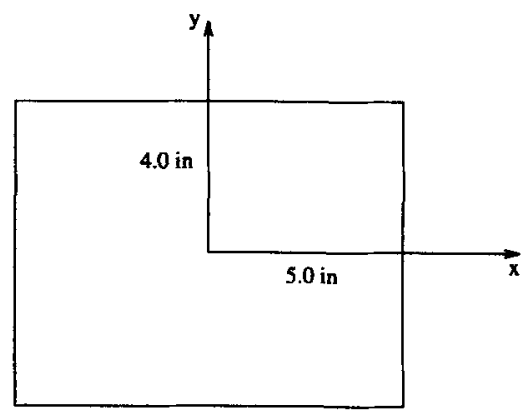

Plate geometry

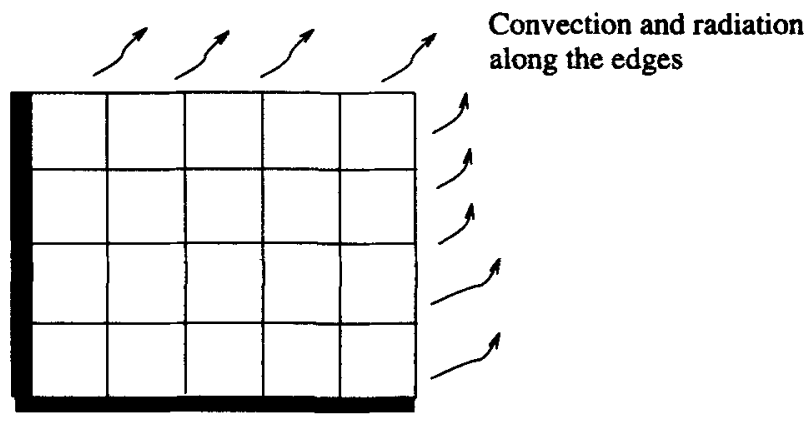



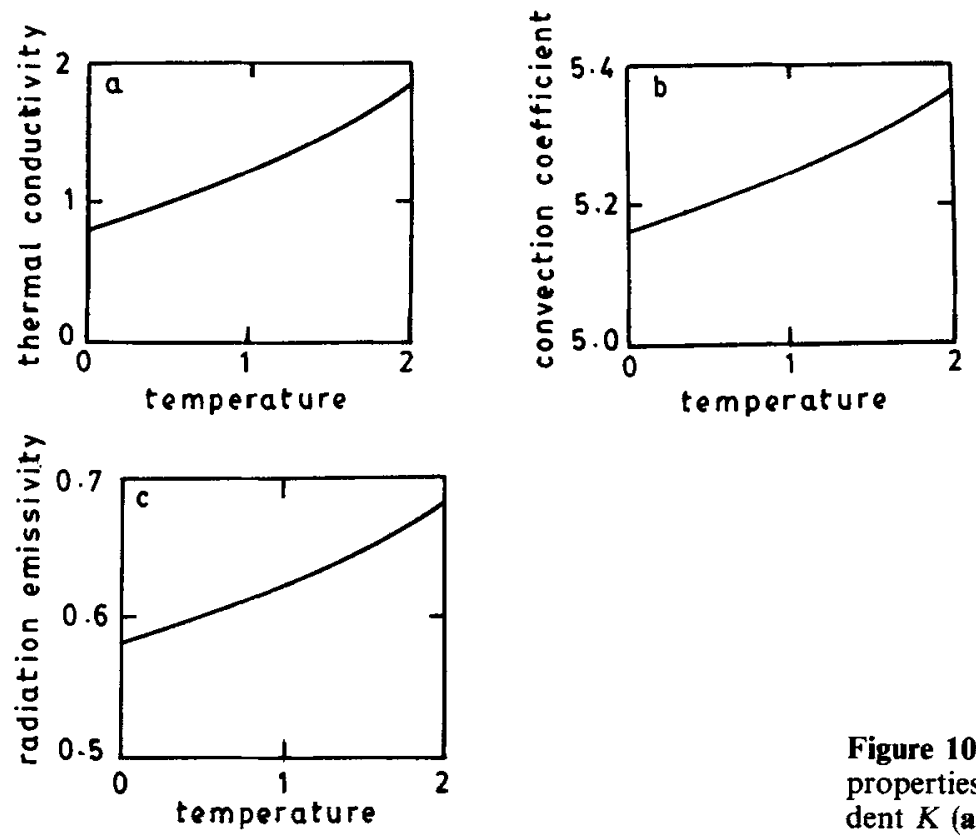

Figure 10. Nonlinear thermal properties. Temperature dependent $K(\mathbf{a}), h(\mathbf{b})$ and $\varepsilon(\mathbf{c})$.

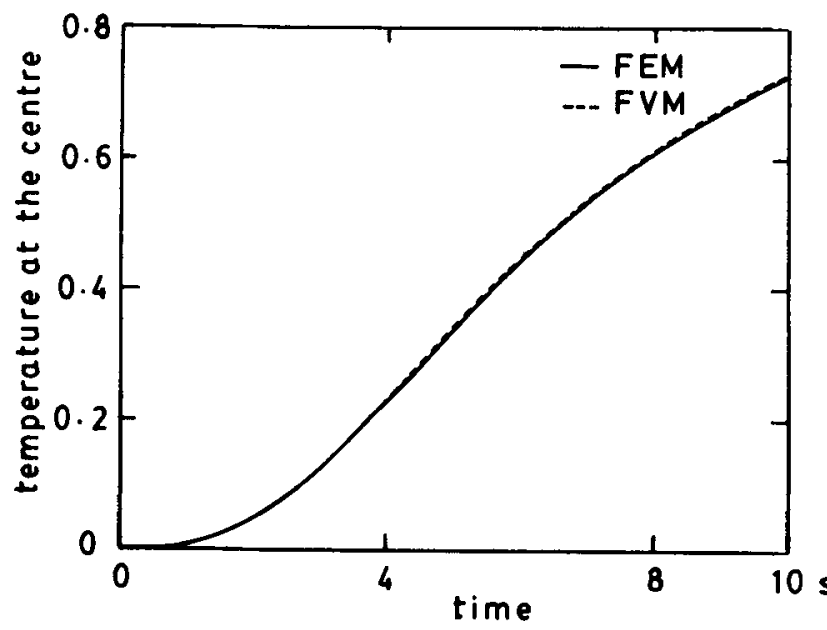

Figure 11. Comparative transient temperature of the centre.

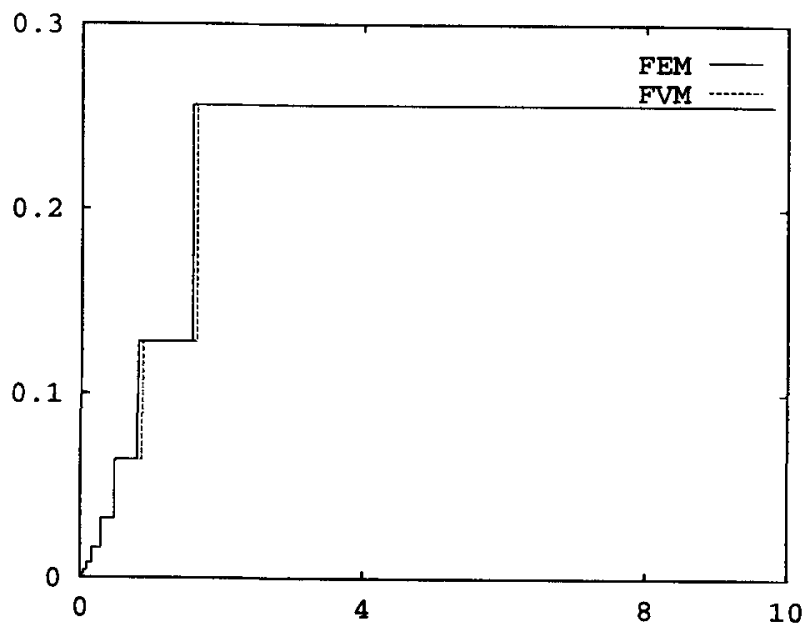

Figure 12. Adaptive time step variations. 

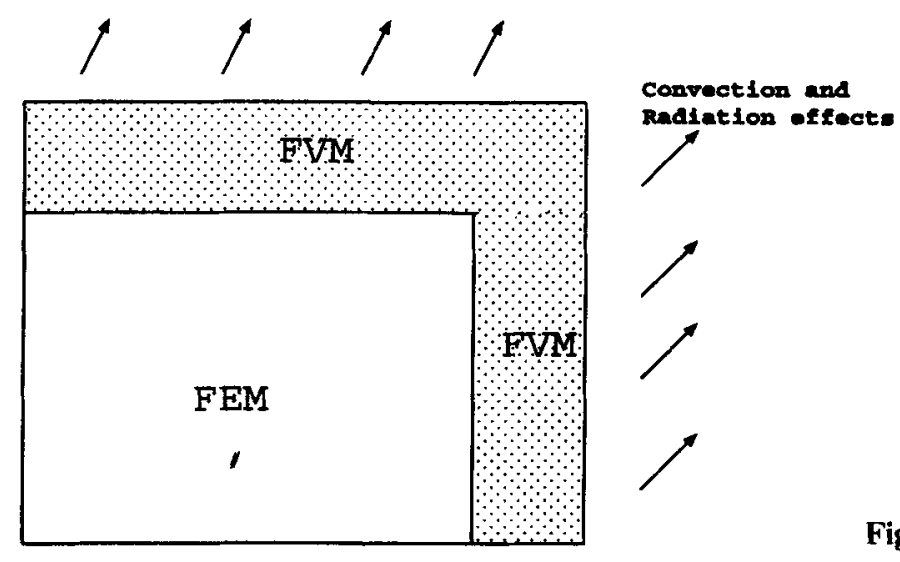

Figure 13. Partitioned meshes.

\section{Mesh partitionings:Finite element and finite volume partitions}

The Galerkin finite element method based on the differential form of the global conservation of energy provides numerical approximations which are in general globally conservative. Such numerical approximations however may violate local conservative properties, which the finite volume type elements preserve, as these have been developed from the local conservation form of the energy equation. Considering the physical situations, with Galerkin finite elements being employed, local conservation is more likely to be violated in regions where there are external heat fluxes acting on the surface of a body. Such cases may lead to solutions which may violate some physical principles (Banaszek 1984). In these instances, the locally conservative finite volume element is cited (Banaszek 1984) to avoid such violations while preserving the local energy conservation. Hence, it is proposed that there be mesh partitionings based on the physical situation to employ locally conservative finite volume elements in the regions where external heat fluxes are present, and to use the Galerkin finite elements over the other regions of the domain. The example problem in which the two sides are subjected to heat fluxes due to convection and radiation is modelled here with these element partitionings. Finite volume elements are used in the regions near to the sides which are subjected to convection and radiation heat fluxes and the remaining region is modelled with Galerkin finite elements. Such a mesh is shown in figure 13, and such a partitioning approach based on two different methodologies will lead to better preservation of local conservation near the external heat flux regions while satisfying global conservation. A time step of 0.05 seconds $(\alpha=0.5)$ was employed in the analysis. The adaptive time step variations for this case are shown in figure 14.

Based on the proposed hypothesis, improved numerical approximations for general heat transfer situations can be obtained by employing mesh partitionings based on the locally conservative finite volume elements in the regions of external fluxes and sudden gradient fluxes to avoid violation of physical laws and preserve local conservation. Galerkin finite elements are employed in other regions. Such modelling and decision-making depends on the intuition, experience and knowledge of the analyst, and currently efforts are underway to establish more rigour for such partitioning strategies. 


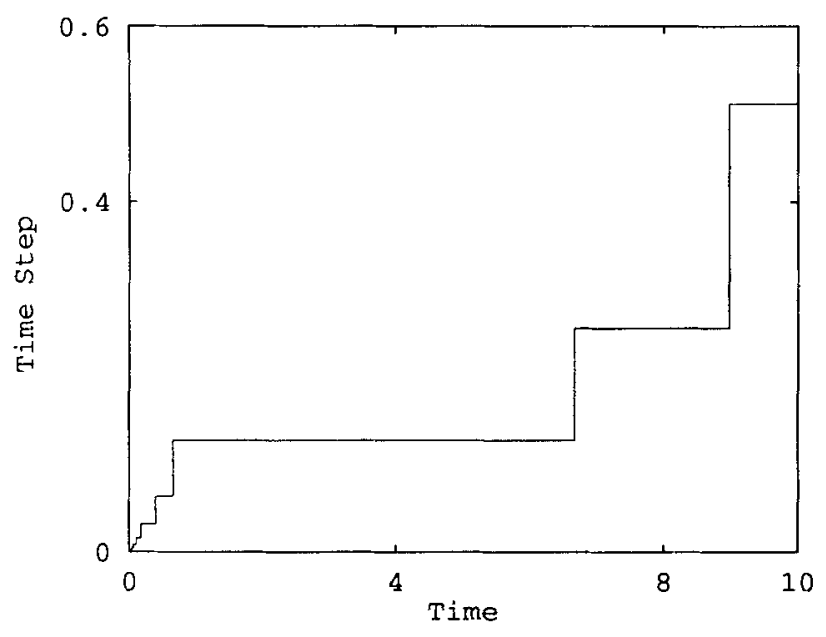

Figure 14. Adaptive time step variations with partitioned meshes.

\section{Concluding remarks}

An adaptive time stepping strategy, based on a posteriori error estimates which permits control of global error for finite element/finite volume computations, was described for applicability to general transient thermal problems. To accurately predict the solution behaviour and to effectively make use of computational resources, such approaches are attractive and involve the use of an optimal number of time steps as opposed to that when a single uniform time stepping is used. Mesh partitioning techniques for combining Galerkin finite elements and finite volume based element approaches were proposed to obtain numerically improved physical representations. Test cases showed that the present formulations can accurately track transient behaviour and that the mesh partitionings provide features for improved numerical representations of the physical situations.

The authors are very pleased to acknowledge support, in part, by NASA-Johnson Space Center/Lockheed Engineering and Space Sciences Co., Houston, Texas, and, by the Army High Performance Computing Research Center (AHPCRC), at the University of Minnesota on a contract from the Army Research Office. Additional support and computing times were furnished by Minnesota Supercomputer Institute at the University of Minnesota. Special thanks are due to Mr Tian Ouyang, University of Minnesota.

\section{References}

Baliga B R, Patankar S V 1983 A control volume finite element method for two dimensional fluid flow and heat transfer. Numer. Heat Transfer 6: 263-282

Banaszek J 1984 A conservative finite element method for heat conduction problems. Int. $J$. Numer. Methods Eng. 20: 2033-2050

Bathe K J 1982 Finite element procedures in engineering analysis (Englewood Cliffs, NJ: Prentice-Hall)

Eriksson K, Johnson C 1987 Error estimators and automatic time step control for nonlinear 
parabolic problems-I. SIAM J. Numer. Anal. 24: 12-23

Hughes T J R 1987 The finite element method (Englewood Cliffs, NJ: Prentice-Hall)

Johnson C 1988 Error estimates and adaptive time-step control for a class of one-step methods for stiff ordinary differential equations. SIAM J. Numer. Anal. 25: 908-926

Lax P D, Wendroff B 1964 Difference schemes for hyperbolic equations with high order accuracy. Commun. Pure Appl. Math. 17: 381

Namburu R R, Tamma K K 1991 Applicability/evaluation of flux based representations for linear/higher order elements for heat transfer in structures: Generalized $\gamma_{T}$-family. In AIAA 29th Aerospace Sciences Meeting, Reno, Nevada

Ouyang T, Tamma K K 1992 On adaptive time stepping approaches for thermal solidification processes. In Proc. ASME Winter Annual Meeting, Anaheim, CA, ASME AMD, vol. 157

Owen D R J, Damjanic F 1983 Reduced numerical integration in thermal transient finite element analysis. Comput. Struc. 17: 261-276

Patankar S V 1980 Numerical heat transfer and fluid flow (New York: McGraw-Hill)

Patankar S V, Baliga B R 1978 A new finite-difference scheme for parabolic differential equations. Numer. Heat Transfer 1: 27-37

Richtmeyer R D, Morton K W 1967 Difference methods for initial value problems. Interscience Tracts in Pure and Applied Mathematics-Number 4 (New York: Interscience)

Schneider G E 1982 Control volume based finite element formulation of the heat conduction equation. In 3rd AIAA/ASME Joint Thermophysics, Fluids, Plasma and Heat Transfer Conference, St. Louis, MO

Schneider G E, Zedan M 1983 Control volume based finite element formulation of the heat conduction equation. Prog. Astronaut. Aeronaut. 86: 305-327

Tamma K K, Namburu 1989 Explicit Lax-Wendroff/Taylor-Galerkin second-order accurate formulations involving flux representations for effective finite element thermal analysis. In AIAA 27th Aerospace Sciences Meeting, Reno, Nevada, AIAA-89-0521

Taylor R L, Zienkiewicz O C, Baynham J M 1983 Mixed and irreducible formulations in finite element analysis. Hybrid and mixed finite element methods. (New York: John Wiley and Sons)

Thomea V, Johnson C, Nie Y 1990 An a posteriori error estimate and adaptive time step control for a backward Euler discretization of a parabolic problem. SIAM J. Numer. Anal. 27: 277-291

Williams S D, Curry D M 1977 An implicit-iterative solution of the heat conduction equation with a radiation boundary condition. Int. J. Numer. Methods Eng. 11: 1605-1619

Zienkiewicz O C, Cheung Y K 1965 Finite elements in the solution of field problems. The Engineer: 507-510 